Article,

\title{
Phenylbutyrate ameliorates high-fat diet-induced obesity via brown adipose tissue activation
}

\author{
Byong-Keol Min ${ }^{1,2,7}$, Hyeon-Ji Kang ${ }^{3,7}$, Byung-Jun Choi ${ }^{1,2}$, Yong Hyun Jeon ${ }^{4,5}$, Je-Yoel Cho ${ }^{6}$, \\ In-Kyu Lee ${ }^{1,3,5^{*}}$ and Dong Wook Kim ${ }^{5,6^{*}}$ \\ 1 Department of Biomedical Science, Graduate School, Kyungpook National University, Daegu, South \\ Korea; minbgj@naver.com (B.-K.M); blackdragon0907@gmail.com (B.-J.C) \\ 2 BK21 plus KNU Biomedical Convergence Programs, Kyungpook National University, Daegu, South Korea \\ 3 Research Institute of Aging and Metabolism, School of Medicine, Kyungpook National University, Daegu, \\ South Korea; hyun13829@hanmail.net (H.J.K) \\ 4 Laboratory Animal Center, Daegu-Gyeongbuk Medical Innovation Foundation (DGMIF), Daegu, South \\ Korea; jeon9014@gmail.com (Y.H.J) \\ 5 Leading-edge Research Center for Drug Discovery and Development for Diabetes and Metabolic Disease, \\ Kyungpook National University, Daegu, South Korea ; leei@knu.ac.kr (I.-K.L) \\ 6 Department of Biochemistry, College of Veterinary Medicine, Seoul National University, 1 Gwanak-ro, \\ Gwanak-gu, Seoul, South Korea; jeycho@snu.ac.kr (J.-Y.C); bellocan@snu.ac.kr (D.W.K) \\ 7 These authors contributed equally to this work \\ * Correspondence: leei@knu.ac.kr; Tel.: +82-53- 420-5564 ; bellocan@snu.ac.kr; Tel: +82-2-880-1288
}

\begin{abstract}
Obesity, which is characterized by an excessive accumulation of body fat, is one of the critical factors causing metabolic syndrome. Many studies have been performed to identify appropriate agents to control obesity, but toxicity remains a problem. Herein, we identified that phenylbutyrate (PBA), which has been used to treat urea cycle disorder with very low toxicity for a long time, efficiently inhibited high fat-induced body weight gain in a diet-induced obesity mouse model (DIO model). PBA treatment decreased body fat mass and increased lean composition. Moreover, PBA increased brown adipose tissue (BAT) activity by increasing glucose uptake, thereby improving glucose tolerance and insulin tolerance. Interestingly, PBA could induce the expression of phosphofructokinase (PFKL), a key enzyme in the glycolytic pathway, and knocking down PFKL dramatically repressed the expression level of Ucp1 as well as those of Prdm16, Cidea, Pgc1 $\alpha$, and Ppar $\gamma$, which are marker genes for BAT activation. These results strongly suggested that PBA could increase energy expenditure by increasing BAT activity via the induction of PFKL. Taken together, PBA could be used as a therapeutic agent for people with obesity to prevent the development of metabolic syndrome.
\end{abstract}

Keywords: Phenylbutyrate, PFKL, Obesity, UCP1, Brown adipose tissue

\section{Introduction}

The imbalance between energy expenditure and food intake is a major factor in obesity, which is characterized by increased body fat accumulation. Adipose tissue is a functional organ that regulates energy homeostasis by alternating between energy storage in the form of triglycerides and energy supply in the form of free fatty acids, depending on the energy status of the body. Continued energy intake stimulates fat cells to proliferate to store excess energy and makes the cells enlarged, leading to inflammation in fat tissue.

Recent studies on overcoming obesity have focused on activating signaling pathways of fat consumption, in which generated energy is dissipated as heat. UCP1 is a well-known key molecule that dissipates energy as heat by uncoupling protons in the inner membrane of mitochondria. UCP1 is most abundant in brown adipose tissue (BAT), which is an organ specialized to consume excess energy as heat. In addition, recent studies have identified that UCP1 expression is inducible in white adipose tissue (WAT) upon cold exposure and beta-adrenergic receptor agonist treatment, converting 
the basic function of WAT from fat storage tissue to fat consumption tissue called beige fat. In either case, UCP1 is indispensable for the consumption of excess energy. The discovery of functional BAT in human adults has resulted in the explosion of BAT studies, and BAT has been identified as a promising target to treat patients with obesity-related disease. UCP1-ablated mice showed an obesity phenotype even when fed a control diet and did not induce diet-induced thermogenesis [1]. In contrast, UCP1 transgenic mice driven by aP2 promoter showed resistance to diet-induced obesity $[2,3]$.

Phenylbutyrate (PBA) has been used to treat urea cycle disorder, which is a genetic disorder caused by a deficiency of one of the enzymes in the urea cycle. Its application has been expanded to various diseases, such as malignancies [4-6], cystic fibrosis [7] and sickle cell anemia [8]. As a histone deacetylase inhibitor, PBA has recently been shown to ameliorate ER stress-mediated metabolic disease [9]. PBA improved obesity-induced chronic inflammation in adipose tissue [10] and protected against liver ischemia reperfusion injury by inhibition of ER stress [11] and liver steatosis by inhibition of de novo lipogenesis [12]. Additionally, PBA has been tested in patients with spinal muscular atrophy and shown beneficial effects [13], indicating that PBA has various beneficial effects on metabolic organs.

In this study, we demonstrate that PBA ameliorates high-fat diet-induced obesity by increasing the activities of brown adipose tissue. Therefore, PBA could be a therapeutic agent for patients with obesity-related disease by targeting brown adipose tissue.

\section{Results}

\subsection{Phenylbutyrate ameliorates HFD-induced obesity}

To see whether PBA has an effect on high-fat diet-induced body weight gain, 8-week-old mice were fed HFD (60\% fat) and LFD (10\% fat) for 8 weeks, and then PBA was administered for an additional 8 weeks with a vehicle control while being fed HFD and LFD. As expected, HFD dramatically induced body weight gain as mice aged, but PBA treatment strongly prevented and showed resistance to HFD-mediated obesity. In addition, body weight gains in the group fed LFD were also slightly but significantly reduced by PBA treatment (Figure 1A), implying that PBA has a therapeutic effect on HFD-induced obesity. To determine which tissues have a dramatic change in weight, we first performed a computed tomography (CT) scan with a live mouse. As shown in Fig $1 \mathrm{~B}$, the most dramatic change in tissue distribution was observed in fat tissue (blue color). The CT scan showed that the dramatic fat accumulation induced by HFD was evidently decreased by treatment with PBA (Figure 1B). Further analysis of body composition showed that PBA treatment decreased fat mass by $9 \%$ (from $51.16 \pm 0.67, \mathrm{~N}=9$ to $47.01 \pm 0.94, \mathrm{~N}=10$ ), while lean body mass was increased by $8 \%$ (from $49.19 \pm 0.79, \mathrm{~N}=9$ to $53.33 \pm 1.9, \mathrm{~N}=10$ ) in the HFD group. Interestingly, PBA treatment to LFD group showed similar effect to that observed in HFD group (Figure 1C).

Next, we tried to figure out whether PBA has an effect on other metabolically active organs such as BAT, liver and WAT. Interestingly, we found that the organ weight of brown adipose tissue was lower and the color was more brownish with PBA treatment than with vehicle in the HFD group, indicating that PBA decreases lipid accumulation by HFD in BAT. The color of the liver was also improved from pale to dark brown by PBA administration, which is similar to the normal phenotype and consistent with previous studies showing that PBA ameliorates liver steatosis [13]. As expected, there was smaller subcutaneous fat tissue (sWAT) in the group treated with PBA than in the group treated with vehicle. Unexpectedly, the size of the epididymal fat tissue (eWAT) was larger in the group treated with PBA than in the vehicle control in HFD group. However, the color of the eWAT was more yellowish in the group fed HFD than in the group fed HFD + PBA, indicating that there is a tissue dysfunction which is caused by accumulated fat with severe tissue inflammation and fibrosis by long-term HFD (Figure 1D), which is consistent with previous report [14]. This observation prompted us to examine tissue histology. Dissected eWAT tissue were subjected to hematoxylin and eosin (H\&E) staining. Interestingly, we did not see a difference in the size of adipocytes between the HFD and HFD + PBA groups, but there was a dramatic increased number of crown-like structure (CLS), marked with a black arrow in eWAT in the group fed HFD with vehicle. In contrast, PBA 
treatment completely blocked the formation of CLS (Figure 1E). As well as eWAT, HFD-induced lipid accumulation either in sWAT and liver was reduced by PBA treatment compare to vehicle treated HFD group (Supplementary Figure S1). Taken together, these results strongly suggested that PBA has beneficial effects on metabolically active organs.

A

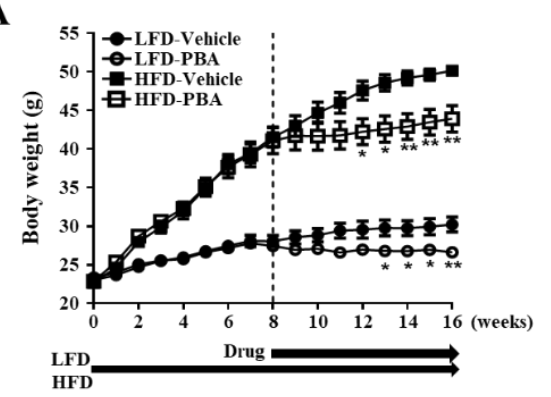

C

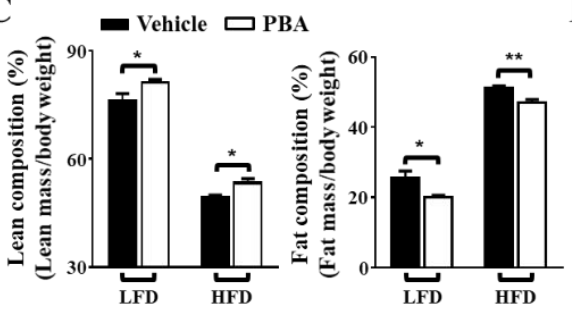

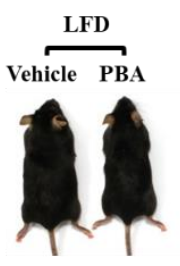

D
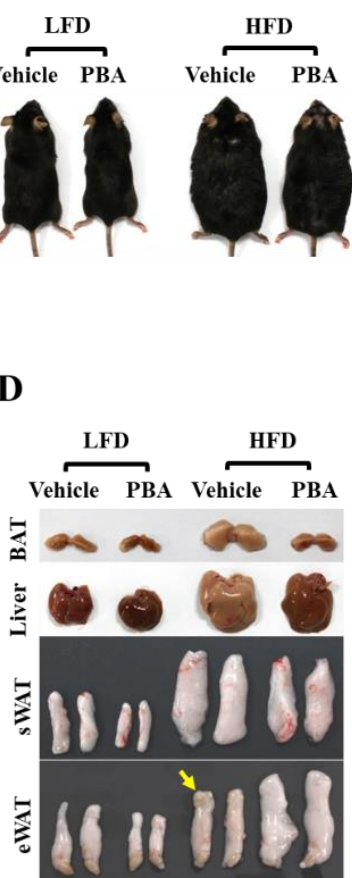

B

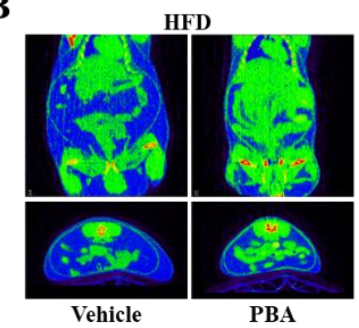

$\mathbf{E}$

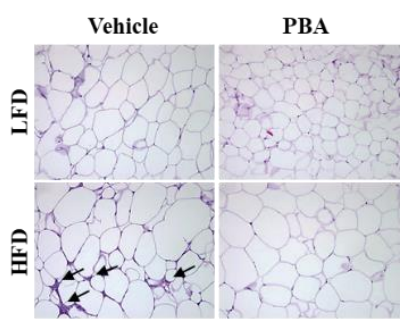

Figure 1. PBA ameliorates HFD-induced obesity. (A) Mouse at the age of 8 weeks fed with LFD and HFD with or without PBA as depicted in the figure. During diet feeding, body weight was monitored in every weeks (left). A representative mouse is shown (right); $n=5$ per LFD and $n=6-7$ per HFD group. (B) CT scanning was performed and fat tissue was indicated as blue color. (C) Body composition analysis was performed to compare fat and lean mass between the HFD and HFD + PBA groups fed LFD or HFD; $\mathrm{n}=8$ per LFD, $\mathrm{n}=9$ per LFD+PBA, $\mathrm{n}=9 \mathrm{HFD}$, and $\mathrm{n}=10$ per HFD + PBA group. (D) BAT, liver, sWAT and eWAT were compared. (E) Tissue sections of eWAT from mice fed LFD or HFD for 24 weeks with or without PBA treatment were subjected to hematoxylin and eosin (H\&E) staining, and the arrows indicate crown-like structures (CLS). Representative pictures are presented. Magnification: X 400. ${ }^{*}$ p-value $<0.05$, and ${ }^{* *}$-value $<0.01$.

\subsection{PBA increases $B A T$ activity}

We hypothesized that the beneficial effect of PBA on HFD-induced mice may be due to the active disposal of glucose in blood and increased energy consumption. To confirm our hypothesis, we tested the effect of PBA on BAT activity, because BAT is well known as an active organ for taking up glucose from blood and converting it into heat and thereby controlling the energy level in the body. BAT from mice fed either LFD or HFD were subjected to H\&E staining. As expected, HFD dramatically increased the formation of lipid droplets, but PBA treatment definitely decreased the fat amount in BAT (Figure 2A). However, we could not make a decision whether the fat formation decreased by PBA is due to the increased BAT activity or other factors. To clarify which aspect is responsible, we measured in vivo glucose uptake using 18F-fluorodeoxyglucose (18F-FDG) positron emission tomography/computed tomography (PET/CT). Interestingly, the BAT activity decreased by HFD was significantly increased by PBA treatment (Figure 2B). In line with these results, PBA significantly decreased fasting glucose levels (Figure $2 \mathrm{C}$ ) and improved glucose tolerance and insulin tolerance compare to either LFD or HFD with vehicle treated group (Figure 2D,E). These results indicate that the decreased fat amount in BAT is due to the increased BAT activity by the PBA treatment, which thereby improves the whole body metabolic phenotype systematically. 
A

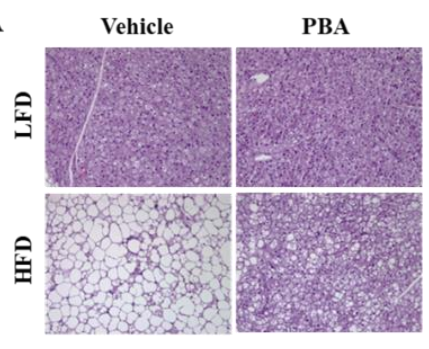

B

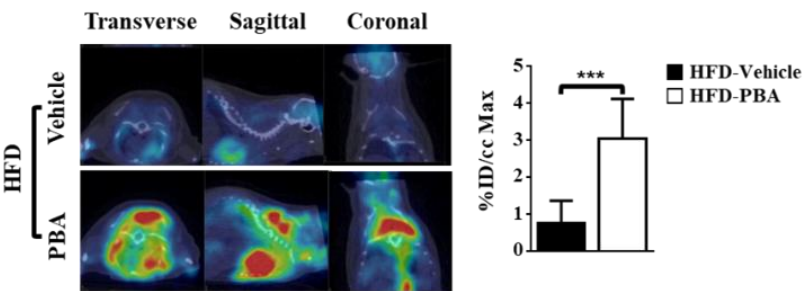

C

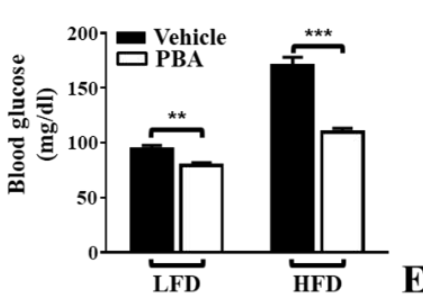

D

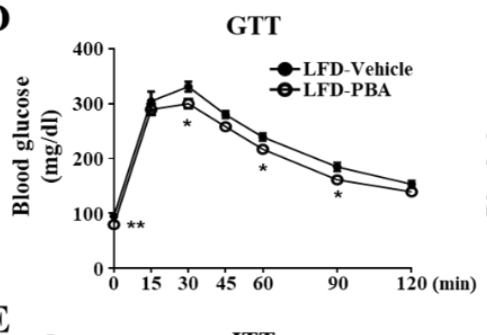

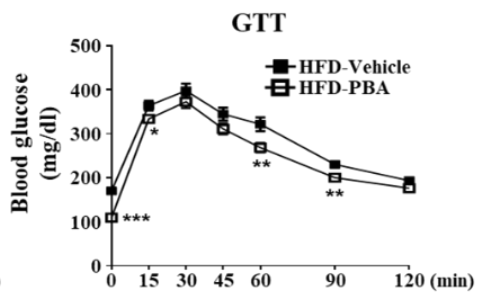

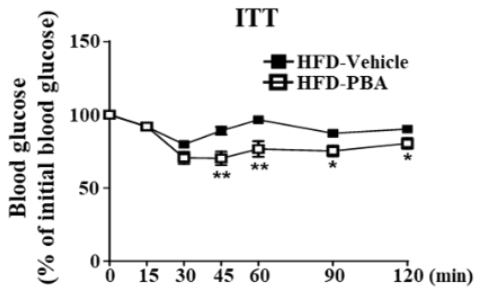

Figure 2. PBA activates BAT. (A) Brown adipose tissue from mice fed LFD or HFD for 16 weeks were dissected, and H\&E staining was performed. Magnification: X 400. (B) PET/CT using 18F-FDG was performed using mice fed HFD for 16 weeks (left), and a quantitative analysis was performed (right). $\mathrm{n}=5$ per each group. (C) Fasting blood glucose levels were measured in mice fasted for $16 \mathrm{~h}$; $\mathrm{n}=8-9$ per LFD and n=12-13 per HFD group. (D) GTT was conducted with mice fasted for $16 \mathrm{~h}$. Glucose (1.5 $\mathrm{g} / \mathrm{kg}$ ) was administered by an i.p. injection. Blood glucose was measured at the indicated time points; $\mathrm{n}=8-9$ per LFD and $\mathrm{n}=12-13$ per HFD group. (E) ITT was performed with mice fasted for $6 \mathrm{~h}$. Insulin $(0.75 \mathrm{U} / \mathrm{kg})$ was administered by an i.p. injection. Blood glucose was measured at the indicated time points; $\mathrm{n}=6-8$ per LFD and $\mathrm{n}=11-12$ per HFD group. ${ }^{*}$-value $<0.05,{ }^{* *} \mathrm{p}$-value $<0.01$, and ${ }^{* * *}$-value $<$ 0.001 .

\subsection{Energy expenditure is increased through PBA-induced BAT activation}

It is previously reported that BAT activation is followed by increased whole body energy expenditure and it results in decreased body fat and obesity $[15,16]$. Our study demonstrates that PBA treatment decreased HFD-induced body weight gain and fat accumulation accompanying with BAT activation. Therefore, we tried to figure out whether PBA-induced BAT activation leads to energy expenditure augmentation. PBA treatment increased $\mathrm{VO}_{2}, \mathrm{VCO}_{2}$ and energy expenditure compared to vehicle treated group under LFD feeding, especially during dark cycle. HFD feeding decreased $\mathrm{VO}_{2}, \mathrm{VCO}_{2}$ and energy expenditure compared to corresponding LFD feeding group, but PBA treatment recovered all these parameters in both light and dark cycle (Figure 3A-C). Physical activity was not altered among all experimental animal groups (Figure 3D). It suggests that PBA improves metabolic processes without alteration in physical activity. 
$\mathbf{A}$

B
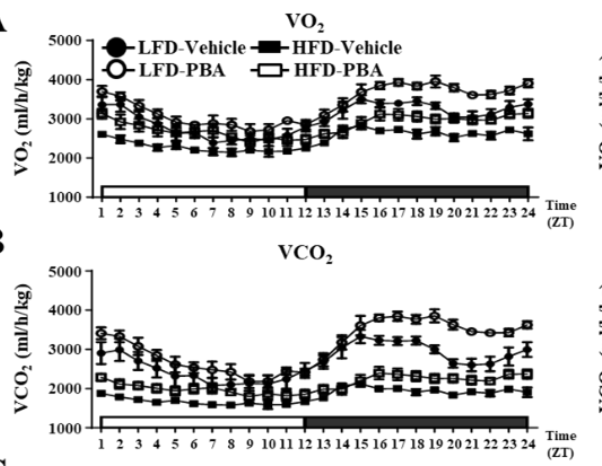

C

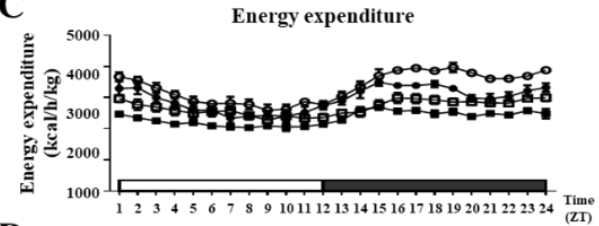

D

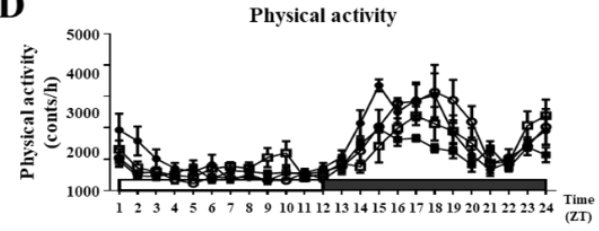

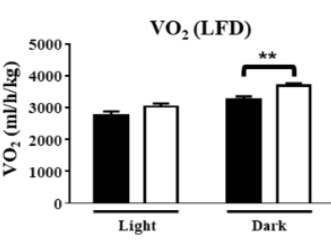

$\mathrm{VCO}_{2}(\mathrm{LFD})$
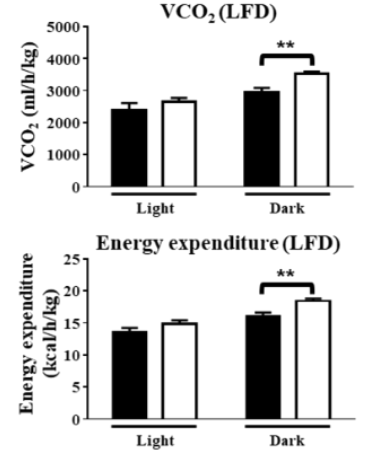

Physical activity (LFD)

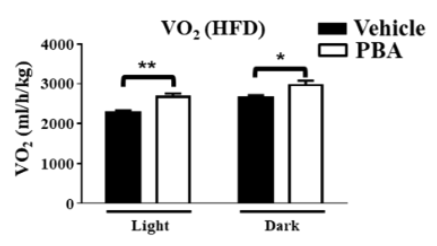

$\mathrm{VCO}_{2}(\mathrm{HFD})$
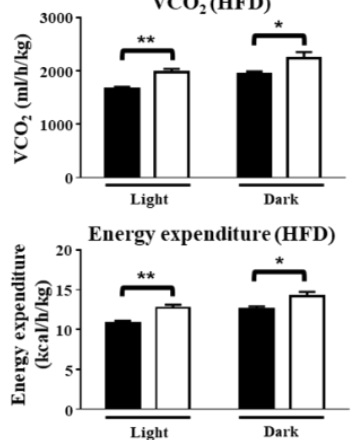

Physical activity (HFD)
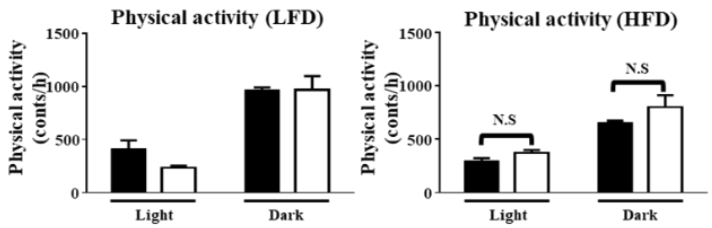

Figure 3. PBA increases energy expenditure. (A-D) The metabolic parameters of mice were determined by metabolic phenocages. $\mathrm{VO}_{2}(\mathbf{A}), \mathrm{VCO}_{2}(\mathbf{B})$, energy expenditure (C) and physical activity (D) were measured and it was represented by light cycle and dark cycle as separately. $n=5$ per each group. ${ }^{*}$ p-value $<0.05,{ }^{* *}$-value $<0.01$, and N.S; nonsignificant.

\section{4. $P F K L$ is required for $P B A$ induced $B A T$ activation}

Using immortalized brown adipocyte (IBA) cells, we tested whether PBA increased glucose uptake. As shown in Figure 4A, PBA significantly increased glucose uptake measured with 18FFDG (Figure 4A). Next, we tried to measure the expression level of the marker genes of BAT activation such as Ppar $\gamma, \operatorname{Pgc} 1 \alpha, \operatorname{Prdm} 16$, and Cidea. We found that PBA treatment significantly increased the transcript levels of Pgc1 $\alpha$, Prdm16, and Cidea but not Ppar $\gamma$, indicating that PBA can increase BAT activity (Figure 4B). To gain insight into the underlying mechanism by which PBA increases glucose uptake, we checked the protein expression levels involved in glycolytic pathways. The expression levels of most proteins were slightly decreased or not altered by long-term treatment with PBA, but PFKL showed a gradually increased expression pattern (Figure 4C). Interestingly, the mRNA levels of Ucp1, Prdm16, Cidea, Pgc1 $\alpha$, and Ppar $\gamma$ were dramatically decreased when PFKL was knocked down using specific siRNA compared with control siRNA (Figure 4D). Taken together, these results strongly indicate that PBA increases energy expenditure by increasing UCP1 through the induction of PFKL expression. 
A

C

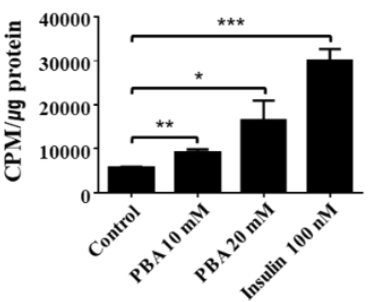

C

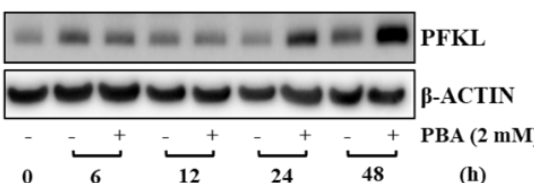

B
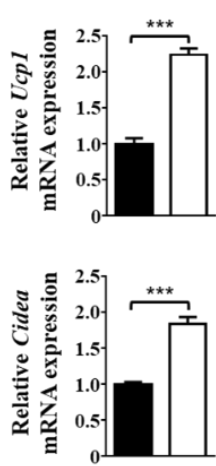

D
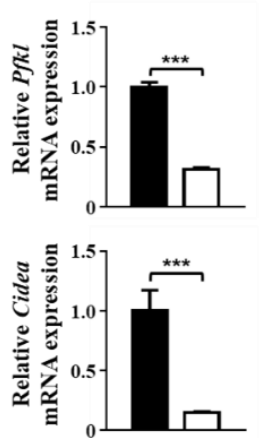
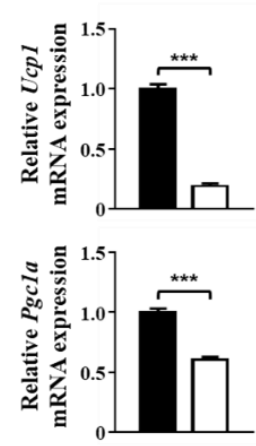

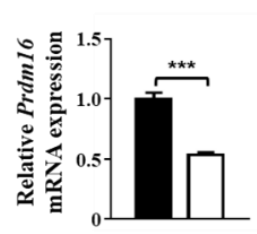

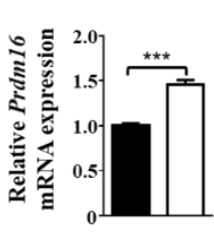
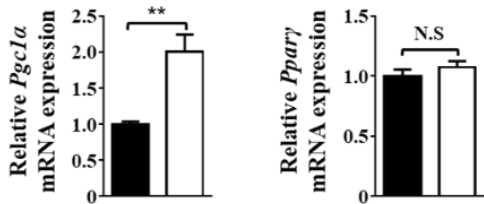

Figure 4. PBA increases genes related to BAT activation and glycolysis related enzyme, PFKL. (A) In vitro glucose uptake measurements using 18F-FDG were performed using immortalized brown adipocyte (IBA) cells. PBA was administered for $30 \mathrm{~min}$ before analysis. Insulin was used as a positive control. $\mathrm{n}=3$ per each group. (B) Genes related to mitochondrial biogenesis were measured in primarycultured BAT cells after treatment with PBA for $12 \mathrm{~h}$ at a dose of $2 \mathrm{mM}$. $\mathrm{n}=3$ per each group. qPCR was performed with specific primers as described in the Material and Methods section. (C) The protein expression level of PFKL was examined by western blot analysis with samples extracted from primary-cultured BAT cells after PBA treatment at the indicated times. PBA was administered at a dose of $2 \mathrm{mM}$. (D) Silencing of PFKL using siRNA decreased the expression level of genes related to mitochondrial activity in primary-cultured BAT cells. The mRNA levels of Ucp1, Prdm16, Cidea, Pgc1 $\alpha$, and Ppar $\gamma$ were measured by qPCR as described in Material and Methods. ${ }^{*}$-value $<0.05$, ${ }^{* *}$ p-value $<0.01,{ }^{* * *}$ p-value $<0.001$, and N.S; nonsignificant.

\section{Discussion}

In this study, we demonstrated that PBA could improve HFD-induced obesity by increasing the activities of BAT. PBA increased the expression level of PFKL, a rate-limiting enzyme in glycolysis, indicating that PBA could stimulate the glycolytic pathway. Moreover, PBA increased the expression of genes related to BAT activation such as Pgc1 $\alpha$, Prdm16, and Cidea transcripts, and UCP1, a key protein that dissipates excess energy as heat in mitochondria. Therefore, PBA could stimulate both the glycolytic pathway and BAT activity. Burning of excess glucose in BAT may give rise to an improved phenotype in other metabolically active organs such as liver and fat tissue. In fact, HFD significantly increased lipid accumulation in both liver and fat tissues, but PBA treatment dramatically decreased fat deposition. Even though the weight of eWAT was greater with PBA treatment than with control treatment in the HFD group, the histology analysis identified that crownlike structures (CLS) were significantly decreased in the group treated with PBA. This result indicates that PBA increases the storage capacity of adipocytes for excess energy by increasing fat size and mass without cellular stress. However, further analysis may need to address the unexpected observation that eWAT mass was increased in spite of the decrease in total fat mass by PBA through the comparing other major fat tissues such as retroperitoneal depots and the mesenteric depots. 
BAT is one of the major organs that burns excess energy in mitochondria. UCP1 is a central key player in that process. Here, we found that PBA treatment increased UCP1 expression in primary cultured cells, strongly suggesting that PBA-mediated UCP1 induction is a major pathway to decrease the blood glucose level. It is likely that UCP1 expression is transcriptionally regulated by PBA, because the UCP1 mRNA level is highly induced by PBA treatment in primary cultured cells. Even though it is not clear how PBA could increase UCP1 mRNA expression in BAT and what is the role of PFKL in PBA mediated UCP1 induction, a recent study may explain a possible regulation of PBA on UCP1 expression via crosstalk between HDAC and H3 lysine 27 residue [17] since PBA has known as a histone deacetylase (HDAC) inhibitor. Further studies needed to address PFKL involvement in PBA mediated UCP1 induction during BAT activation.

PFK1 (phosphofructokinase 1) converts fructose 6-phosphate and ATP into fructose 1,6bisphosphate and ADP. Since PFK1 induces ATP-dependent phosphorylation to convert fructose 6phosphate during glycolysis, it is regarded as one of the rate-limiting enzymes in glycolysis. In this study, we found that the protein expression level of PFKL, one of the isoforms of PFK1, was dramatically increased by PBA treatment. These data strongly suggested that PBA may stimulate the rate-limiting step in glycolysis by upregulating the PFKL enzyme, thereby increasing glucose uptake. Increased PFKL expression by PBA may be occurring at the transcription level because PBA has an inhibitory effect on HDAC. However, further studies need to identify the detailed mechanisms. In addition, knockdown of PFKL in BAT cells suppressed BAT activation related genes. It demonstrated that increased PFKL expression by PBA could regulate BAT activation related genes. Somehow it is unclear whether PFKL induction is essential for PBA-induced BAT activation or it has just a role for that as partly.

In conclusion, we demonstrated that PBA evidently increases BAT activity and ameliorates HFD-induced obesity. Furthermore, PBA induced several genes related to BAT activation, such as Pgc1 $\alpha$, Prdm16, and Cidea as well as Ucp1, indicating that PBA stimulates. Taken together, our data suggest that PBA is a potential therapeutic agent for patients with obesity.

\section{Materials and Methods}

\subsection{Animals}

Male C57BL/6J mice (8 weeks old) were fed with Low-fat diet (LFD-10\% fat, Research Diets D12450B) or High-fat diet (HFD-60\% fat, Research Diets D12492) for 8 weeks. After that, each group of mice was subjected to gavage every $12 \mathrm{~h}$ with vehicle (distilled water) or sodium PBA $(500 \mathrm{mg} / \mathrm{kg}$, Santa Cruz Biotechnology, sc-200652A) [9, 18] for additional 8 weeks while being fed LDF and HFD for monitoring body weight gain. Body weight and food intake were monitored on a weekly basis. The mice were acclimatized to a $12 \mathrm{~h}$ light/dark cycle at $22-24{ }^{\circ} \mathrm{C}$. All animal experiments were performed according to the National Institutes of Health Guide for the Care and Use of Laboratory animals (NIH Publication no. 8023, revised 1978)

\subsection{In vivo PET/CT analysis and in vitro glucose measurement using 18F-fluorodeoxyglucose (18F-FDG)}

Mice were administered intravenously with 7.4 MBq of 18F-FDG, and then a 20-min scan was performed with a Triumph II PET/CT system (LabPET8, Gamma Medica-Ideas Inc., Waukesha, WI) at 30-60 min post injection. A CT scan was done with an X-ray detector right after the acquisition of PET imaging. PET images were reconstructed by 3D-OSEM iterative image reconstruction, and reconstructions for $\mathrm{CT}$ images were performed using filtered back-projections. All mice were anesthetized using 1-2\% isoflurane gas during imaging. Functional PET images were coregistered with anatomical CT images using 3D image visualization and analysis software VIVID (Gamma Medica-ideas, Northridge, CA). The uptake $(\% \mathrm{ID} / \mathrm{cc})$ for the volumes of interest (VOIs) from each image were determined by manually drawing the brown adipose tissue of interest in coregistered CT images using PMOD 3.5 software (PMOD Technologies, Zurich, Switzerland).

For in vitro glucose measurement using 18F-FDG, immortalized brown adipocyte (IBA) cells were seeded in a 24-well plate and differentiated. For measurement of FDG uptake, the differentiated 
IBAs were incubated with DMEM 5.5 mM glucose containing 0.5\% FBS medium for $12 \mathrm{~h}$. The medium was changed to Krebs-Ringer HEPES (KRH) buffer (50 mM HEPES, $137 \mathrm{mM} \mathrm{NaCl}, 4.7 \mathrm{mM} \mathrm{KCl}, 1.85$ $\mathrm{mM} \mathrm{CaCl} 2,1.3 \mathrm{mM} \mathrm{MgSO}$, and $0.1 \% \mathrm{BSA}$ ) for $2 \mathrm{~h}$, and the chemicals were administered for $30 \mathrm{~min}$.

\subsection{Body composition analysis}

After sodium PBA administration for 10 weeks, fat mass and lean body mass were measured and analyzed by nuclear magnetic resonance using a Bruker Mouse Minispec (Bruker-LF50 Model) following the manufacturer's instructions.

\subsection{Glucose tolerance tests and insulin tolerance test}

For the glucose tolerance test (GTT), the mice that were administered sodium PBA for 8 weeks were fasted for $16 \mathrm{~h}$, then received D-glucose $(1.5 \mathrm{~g} / \mathrm{kg})$ via an i.p. injection. Additional sodium PBA was treated $2 \mathrm{~h}$ before measurement. For the insulin tolerance test (ITT), the mice that were administered sodium PBA for 9 weeks were fasted for $6 \mathrm{~h}$, then received insulin $(0.75 \mathrm{U} / \mathrm{kg})$ via an i.p. injection. Additional sodium PBA was treated $2 \mathrm{~h}$ before measurement. Blood glucose levels were measured by an ACCU-CHECK Active blood sugar test meter (Roche) at the indicated time points $(0,15,30,45,60,90$ and $120 \mathrm{~min})$.

\subsection{Total $m R N A$ extraction and quantitative $R T-P C R$}

Total RNA from cells was extracted using QIAzol reagent (Qiagen, Valencia, CA, USA) and cDNA was synthesized using a RevertAidTM First Strand cDNA Synthesis kit (Fermentas, Vilnius, Lithuania) according to the manufacturer's instructions. Quantitative Real-time PCR was performed using SYBR Green (SYBR Green Master Mix, Applied Biosystems, Warrington, UK) with a StepOneTM Real-Time PCR system (Applied Biosystems). The primers used were as follows: UCP1, forward: 5'-GGTCGTGAAGGTCAGAATGCA-3', reverse: 5'-GCATTGTAGGTCCCCGTGTAG-3'; PGC1a, forward: 5'-TGCGGGATGATGGAGACA-3', reverse: 5'-GCGAAAGCGTCACAGGTGTA3'; PRDM16, forward: 5'-AACCAGGCATCC ACTCGAAT-3', reverse: 5' TGGACACGGTGGGTTGCT-3'; Cidea, forward: 5'-TGACCC CCCTCATACATCCA-3', reverse: 5'GGCTACTTCGGTCATGGTTTG-3'; PPAR $\gamma$, forward: 5'- CACAAGAGCTGACCCAATGGT-3', reverse: 5' ${ }^{\prime}$ GATCGCACTTTGGTATTCTTGGA $\quad 3^{\prime} ; \quad 36 \mathrm{~B} 4$, forward: 5'ACCTCCTTCTTCCAGGCTTT-3', reverse: 5'-CTCCAGTCTTTAT CAGCTGC-3'.

\subsection{Metabolic phenocages}

To measure the metabolic parameters, which included VO2, VCO2, and physical activity, the mice were fed LFD or HFD for 21 weeks with vehicle (distilled water) or PBA sodium PBA (500 $\mathrm{mg} / \mathrm{kg}$, twice/day by oral gavage) for last 13 weeks. After then the mice were housed individually in an indirect calorimetric cage (TSE PhenoMaster) as previously reported [19]. Each mouse was monitored during $48 \mathrm{~h}$ in the fed state and all results were analyzed for $24 \mathrm{~h}$ on average. The energy expenditure was calculated using $\mathrm{VO}_{2}$ and $\mathrm{VCO}_{2}$.

\subsection{Primary culture from $B A T$}

The interscapular brown adipose tissues of 3- to 4-week-old mice were minced in a collagenase buffer and digested at $37^{\circ} \mathrm{C}$ at $160 \mathrm{rpm}$ for $25 \mathrm{~min}$. The digested fat tissue was filtrated through a 100$\mu \mathrm{m}$ cell strainer and centrifuged at $1500 \mathrm{rpm}$ for $5 \mathrm{~min}$. The supernatant was removed, and pellet containing the stromal vascular fraction cells was filtrated through a $40-\mu \mathrm{m}$ cell strainer. After centrifugation, the pellet was plated in high glucose-DMEM supplemented with $10 \%$ bovine serum. For brown adipocyte differentiation, confluent precursor cells were incubated in medium supplemented with $5 \mu \mathrm{g} / \mathrm{ml}$ insulin, $0.5 \mu \mathrm{M}$ dexamethasone, $0.25 \mathrm{mM}$ IBMX, $2 \mathrm{nM}$ T3, $0.065 \mathrm{mM}$ indomethacin, $1 \mu \mathrm{M}$ rosiglitazone and 10\% fetal bovine serum. After 2 days, the medium was replaced with medium supplemented with $1 \mu \mathrm{g} / \mathrm{ml}$ insulin, $2 \mathrm{nM}$ T3 and 10\% FBS and then changed every 2 days with medium containing 10\% FBS and $2 \mathrm{nM}$ T3. 


\subsection{Western blot analysis}

Total cell lysates were prepared using lysis buffer containing $20 \mathrm{mM}$ Tris (pH 7.4), $5 \mathrm{mM}$ EDTA (pH 8.0), $10 \mathrm{mM} \mathrm{Na} 4 \mathrm{P} 2 \mathrm{OH}, 100 \mathrm{mM} \mathrm{NaF}, 2 \mathrm{mM} \mathrm{Na} 3 \mathrm{VO} 4,1 \% \mathrm{NP}-40,0.1 \mathrm{mM}$ PMSF, and proteinase and phosphatase inhibitors. The PFKL primary antibodies were purchased from Abcam (Cambridge, UK). Detection of each protein was performed using an ECL western blotting detection system.

\section{9. siRNA transfection}

$70 \%$ confluent primary brown fat precursor cells were transfected with $25 \mathrm{nM}$ siRNA targeting PFKL or nonsilencing control siRNA using Lipofectamine RNAiMAX (Invitrogen, Carlsbad, CA, USA) according to the manufacturer's instructions.

\section{10. Statistical analyses}

Data are expressed as the mean \pm standard error of the mean. Significant differences between groups were calculated using Student's t-test via GraphPad Prism 5 (GraphPad Software, CA). P<0.05 was considered statistically significant.

Supplementary Materials: Supplementary materials can be found at www.mdpi.com/xxx/s1.

Author Contributions: Conception and design of study: B.K.M., H.J.K., D.W.K.; Acquisition of data: B.K.M., H.J.K., B.J.C.; Analysis and interpretation of data: B.K.M., H.J.K., B.J.C., Y.H.J.; Drafting the manuscript: B.K.M., I.K.L., D.W.K.; Writing-Revision \& Editing: J.Y.C., I.K.L., D.W.K.

Funding: This work was supported by the Basic Science Research Program through the National Research Foundation of Korea (NRF) funded by the Ministry of Education (NRF-2014R1A1A2055894, 2017R1D1A1B03030844, and 2017R1D1A1B03028340), by a grant from the Korea Health Technology R\&D Project through the Korea Health Industry Development Institute (KHIDI), funded by the Ministry of Health \& Welfare, Republic of Korea. (Grant Number: HI16C1501), and by the National Research Foundation (NRF) funded by the Ministry of Science, ICT \& Future Planning (2016M3A9B6026771, 2014M3A9D5A01073598)

Conflicts of Interest: The authors declare no conflict of interest. The funders had no role in the design of the study; in the collection, analyses, or interpretation of data; in the writing of the manuscript, or in the decision to publish the results.

\section{Abbreviations}

$\begin{array}{ll}\text { PBA } & \text { Phenylbutyrate } \\ \text { DIO } & \text { Diet-induced obesity } \\ \text { LFD } & \text { Low fat diet } \\ \text { HFD } & \text { High fat diet } \\ \text { BAT } & \text { Brown adipose tissue } \\ \text { eWAT } & \text { epididymal white adipose tissue } \\ \text { sWAT } & \text { subcutaneous white adipose tissue } \\ \text { UCP1 } & \text { Uncoupling protein 1 } \\ \text { PFKL } & \text { 6-phosphofructokinase, liver type } \\ \text { 18F } & \text { Fluorine-18; } 18 \mathrm{~F} \\ \text { FDG } & \text { Fluorodeoxyglucose }\end{array}$

\section{References}

1. Feldmann, H. M.; Golozoubova, V.; Cannon, B.; Nedergaard, J., UCP1 ablation induces obesity and abolishes diet-induced thermogenesis in mice exempt from thermal stress by living at thermoneutrality. Cell metabolism 2009, 9, (2), 203-9. 
2. Kopecky, J.; Clarke, G.; Enerback, S.; Spiegelman, B.; Kozak, L. P., Expression of the mitochondrial uncoupling protein gene from the aP2 gene promoter prevents genetic obesity. The Journal of clinical investigation 1995, 96, (6), 2914-23.

3. Kopecky, J.; Hodny, Z.; Rossmeisl, M.; Syrovy, I.; Kozak, L. P., Reduction of dietary obesity in aP2-Ucp transgenic mice: physiology and adipose tissue distribution. The American journal of physiology 1996, 270, (5 Pt 1), E768-75.

4. Bar-Ner, M.; Thibault, A.; Tsokos, M.; Magrath, I. T.; Samid, D., Phenylbutyrate induces cell differentiation and modulates Epstein-Barr virus gene expression in Burkitt's lymphoma cells. Clinical cancer research : an official journal of the American Association for Cancer Research 1999, 5, (6), 1509-16.

5. Gore, S. D.; Carducci, M. A., Modifying histones to tame cancer: clinical development of sodium phenylbutyrate and other histone deacetylase inhibitors. Expert opinion on investigational drugs 2000, 9, (12), 2923-34.

6. Gilbert, J.; Baker, S. D.; Bowling, M. K.; Grochow, L.; Figg, W. D.; Zabelina, Y.; Donehower, R. C.; Carducci, M. A., A phase I dose escalation and bioavailability study of oral sodium phenylbutyrate in patients with refractory solid tumor malignancies. Clinical cancer research : an official journal of the American Association for Cancer Research 2001, 7, (8), 2292-300.

7. Rubenstein, R. C.; Lyons, B. M., Sodium 4-phenylbutyrate downregulates HSC70 expression by facilitating mRNA degradation. American journal of physiology. Lung cellular and molecular physiology 2001, 281, (1), L43-51.

8. Dover, G. J.; Brusilow, S.; Charache, S., Induction of fetal hemoglobin production in subjects with sickle cell anemia by oral sodium phenylbutyrate. Blood 1994, 84, (1), 339-43.

9. Ozcan, U.; Yilmaz, E.; Ozcan, L.; Furuhashi, M.; Vaillancourt, E.; Smith, R. O.; Gorgun, C. Z.; Hotamisligil, G. S., Chemical chaperones reduce ER stress and restore glucose homeostasis in a mouse model of type 2 diabetes. Science 2006, 313, (5790), 1137-40.

10. Kawasaki, N.; Asada, R.; Saito, A.; Kanemoto, S.; Imaizumi, K., Obesity-induced endoplasmic reticulum stress causes chronic inflammation in adipose tissue. Scientific reports 2012, 2, 799.

11. Vilatoba, M.; Eckstein, C.; Bilbao, G.; Smyth, C. A.; Jenkins, S.; Thompson, J. A.; Eckhoff, D. E.; Contreras, J. L., Sodium 4-phenylbutyrate protects against liver ischemia reperfusion injury by inhibition of endoplasmic reticulum-stress mediated apoptosis. Surgery 2005, 138, (2), 342-51.

12. Ren, L. P.; Song, G. Y.; Hu, Z. J.; Zhang, M.; Peng, L.; Chen, S. C.; Wei, L.; Li, F.; Sun, W., The chemical chaperon 4-phenylbutyric acid ameliorates hepatic steatosis through inhibition of de novo lipogenesis in high-fructose-fed rats. International journal of molecular medicine 2013, 32, (5), 1029-36.

13. Mercuri, E.; Bertini, E.; Messina, S.; Pelliccioni, M.; D'Amico, A.; Colitto, F.; Mirabella, M.; Tiziano, F. D.; Vitali, T.; Angelozzi, C.; Kinali, M.; Main, M.; Brahe, C., Pilot trial of phenylbutyrate in spinal muscular atrophy. Neuromuscular disorders : NMD 2004, 14, (2), 130-5.

14. Altintas, M. M.; Rossetti, M. A.; Nayer, B.; Puig, A.; Zagallo, P.; Ortega, L. M.; Johnson, K. B.; McNamara, G.; Reiser, J.; Mendez, A. J.; Nayer, A., Apoptosis, mastocytosis, and diminished adipocytokine gene expression accompany reduced epididymal fat mass in long-standing diet-induced obese mice. Lipids in health and disease 2011, 10, 198.

15. Lowell, B. B.; Spiegelman, B. M., Towards a molecular understanding of adaptive thermogenesis. Nature 2000, 404, (6778), 652-60.

16. Saito, M., Brown adipose tissue as a therapeutic target for human obesity. Obesity research $\mathcal{E}$ clinical practice $\mathbf{2 0 1 3}, \mathbf{7},(6), \mathrm{e} 432-8$. 
17. Li, F.; Wu, R.; Cui, X.; Zha, L.; Yu, L.; Shi, H.; Xue, B., Histone Deacetylase 1 (HDAC1) Negatively Regulates Thermogenic Program in Brown Adipocytes via Coordinated Regulation of Histone H3 Lysine 27 (H3K27) Deacetylation and Methylation. The Journal of biological chemistry 2016, 291, (9), 452336.

18. Liu, S. H.; Yang, C. C.; Chan, D. C.; Wu, C. T.; Chen, L. P.; Huang, J. W.; Hung, K. Y.; Chiang, C. K., Chemical chaperon 4-phenylbutyrate protects against the endoplasmic reticulum stress-mediated renal fibrosis in vivo and in vitro. Oncotarget 2016, 7, (16), 22116-27.

19. Oh, C. M.; Namkung, J.; Go, Y.; Shong, K. E.; Kim, K.; Kim, H.; Park, B. Y.; Lee, H. W.; Jeon, Y. H.; Song, J.; Shong, M.; Yadav, V. K.; Karsenty, G.; Kajimura, S.; Lee, I. K.; Park, S.; Kim, H., Regulation of systemic energy homeostasis by serotonin in adipose tissues. Nature communications $2015,6,6794$. 